PROCEEDINGS OF THE

AMERICAN MATHEMATICAL SOCIETY

Volume 129, Number 11, Pages 3279-3284

S 0002-9939(01)05977-9

Article electronically published on March 29, 2001

\title{
REVÊTEMENTS ET ISOMÉTRIES POUR LA MÉTRIQUE INFINITÉSIMALE DE KOBAYASHI
}

\author{
JEAN-PIERRE VIGUÉ
}

(Communicated by Steven R. Bell)

\begin{abstract}
In this paper, we prove that, under some hypothesis on the domains, if a holomorphic mapping $f: D_{1} \longrightarrow D_{2}$ is an isometry for the Kobayashi infinitesimal metric at a point, it is a covering map. In the case $D_{1}=D_{2}$, we prove, in certain cases, that $f$ is an analytic isomorphism.
\end{abstract}

\section{INTRODUCTION}

Ces dernières années, un certain nombre d'auteurs (I. Graham [4], L. Belkhchicha [1], J.-P. Vigué [1] et [12]) ont essayé de caractériser les isomorphismes analytiques entre domaines bornés à l'aide des métriques invariantes. Citons en particulier les deux théorèmes suivants.

Théorème 1.1 (J.-P. Vigué [11]). Soient $D_{1}$ et $D_{2}$ deux domaines bornés de $\mathbb{C}^{n}$, et supposons que $D_{1}$ soit convexe. Soit $a \in D_{1}$, et soit $f: D_{1} \longrightarrow D_{2}$ une application holomorphe telle que, pour tout $v \in \mathbb{C}^{n}$,

$$
E_{D_{2}}\left(f(a), f^{\prime}(a) \cdot v\right)=E_{D_{1}}(a, v),
$$

(où $E_{D}$ désigne la métrique infinitésimale de Carathéodory sur D). Alors $f$ est un isomorphisme analytique de $D_{1}$ sur $D_{2}$.

De manière duale, si $D_{2}$ est convexe, il faut utiliser la métrique infinitésimale de Kobayashi, et on a le résultat suivant.

Théorème 1.2 (I. Graham [4]). Soit $M$ une variété complexe taut (au sens de $H$. Wu 13]) de dimension $n$, soit $D$ un domaine strictement convexe borné de $\mathbb{C}^{n}$. Soit $a \in M$ et soit $f: M \longrightarrow D$ une application holomorphe telle que, pour tout $v \in T_{a}(M)$,

$$
F_{D}(f(a), T f(a) \cdot v)=F_{M}(a, v) .
$$

Alors $f$ est un isomorphisme analytique de $M$ sur $D$.

Les démonstrations utilisent un résultat dû à L. Lempert [8] et H. Royden et P. Wong [9] (voir aussi M. Jarnicki et P. Pflug [6]) sur l'égalité des métriques infinitésimales de Carathéodory et de Kobayashi sur un domaine convexe borné de $\mathbb{C}^{n}$. On ne peut donc pas espérer démontrer un résultat semblable lorsque l'un des

Received by the editors March 10, 2000.

2000 Mathematics Subject Classification. Primary 32F45.

(C)2001 American Mathematical Society 
deux domaines n'est pas supposé convexe. D'autre part, on sait aussi (voir par exemple S. Kobayashi [7]) que, si $\pi: D_{1} \longrightarrow D_{2}$ est une application holomorphe et un revêtement, alors $\pi$ est une isométrie pour la métrique infinitésimale de Kobayashi en tout point, c'est-à-dire, que, pour tout point $z \in D_{1}$, pour tout $v \in \mathbb{C}^{n}$,

$$
F_{D_{2}}\left(f(z), f^{\prime}(z) \cdot v\right)=F_{D_{1}}(z, v) .
$$

Comme nous allons le voir maintenant, la métrique infinitésimale de Kobayashi caractérise, du moins dans certains cas, les revêtements. Plus précisément, nous montrerons le théorème suivant.

Théorème 1.3. Soit $M_{1}$ une variété complexe connexe taut de dimension n, soit $M_{2}$ une variété complexe connexe de même dimension $n$ dont le revêtement universel est analytiquement isomorphe à un domaine borné strictement convexe de $\mathbb{C}^{n}$. Soit $a \in M_{1}$ et soit $f: M_{1} \longrightarrow M_{2}$ une application holomorphe telle que, pour tout $v \in T_{a}(M)$,

$$
F_{M_{2}}(f(a), T f(a) \cdot v)=F_{M_{1}}(a, v) .
$$

Alors $f$ est un revêtement de $M_{1}$ sur $M_{2}$.

Nous montrerons également que l'on peut remplacer l'hypothèse que le revêtement universel de $M_{2}$ est isomorphe à un domaine borné strictement convexe par l'hypothèse que les revêtements universels de $M_{1}$ et $M_{2}$ sont tous les deux isomorphes à des domaines bornés convexes.

Nous montrerons ensuite que ces résultats permettent de montrer pour des applications holomorphes de $D$ dans $D$, ce que j'appelerai un lemme de Scharz-Pick. Par exemple, nous montrerons le théorème suivant:

Théorème 1.4. Soit $D$ un domaine borné de $\mathbb{C}$. Supposons qu'il existe une constante $\delta>0$ telle que, pour tout lacet $\gamma$ dans $D$, de classe $\mathcal{C}^{1}$ par morceaux non homotope à un lacet constant, la longueur de $\gamma$ soit supérieure à $\delta$. Soit $a \in D$ et soit $f: D \longrightarrow D$ une application holomorphe telle que, pour tout $v \in \mathbb{C}$, on ait:

$$
F_{D}(f(a), T f(a) \cdot v)=F_{D}(a, v) .
$$

Alors $f$ est un automorphisme analytique de D.

Nous montrerons de même un résultat valable pour certains domaines bornés de $\mathbb{C}^{n}$ en utilisant la longueur des lacets pour la métrique infinitésimale de Kobayashi.

Enfin, nous terminerons ce travail par un certain nombre de questions et d'exemples.

\section{RAPPELS ET PREMIÈRES PROPRIÉTÉS}

La métrique infinitésimale de Carathéodory $E_{D}$ sur une variété complexe $D$ est définie (voir [3], 6] et [7]) par

$$
E_{D}(x, v)=\sup _{f \in H(D, \Delta)}|T f(x) \cdot v|\left(x \in D, v \in T_{x} D\right) .
$$

La métrique infinitésimale de Kobayashi $F_{D}$ est définie de manière duale:

$$
F_{D}(x, v)=\inf \left\{|\lambda| \mid \exists \varphi \in H(\Delta, D) \text { tel que } \varphi(0)=x \text { et } \lambda \varphi^{\prime}(0)=v\right\} .
$$


D'autre part, si $\alpha$ est une métrique infinitésimale sur $D$ et si $\gamma:[a, b] \longrightarrow D$ est un chemin de classe $\mathcal{C}^{1}$ par morceaux, on définit la longueur de $\gamma$ (pour la métrique infinitésimale $\alpha$ ) par la formule:

$$
L_{\alpha}(\gamma)=\int_{a}^{b} \alpha\left(\gamma(t), \gamma^{\prime}(t)\right) d t
$$

En particulier, si on considère $\mathbb{C}^{n}$ muni de la norme hermitienne canonique $\|$.$\| et$ si on prend $\alpha(x, v)=\|v\|$, on notera $L(\gamma)$ la longueur ainsi obtenue.

La longueur $L_{\alpha}(\gamma)$ étant ainsi définie, on peut alors définir une distance intégrée $d_{\alpha}$ sur $D$ de la façon suivante: la distance $d_{\alpha}$ de deux points $x$ et $y$ de $D$ est la borne inférieure des longueurs des chemins de classe $\mathcal{C}^{1}$ par morceaux d'origine $x$ et d'extrémité $y$. En particulier, quand on prend pour $\alpha$ la métrique infinitésimale de Kobayashi $F_{D}$, nous noterons $L_{k}(\gamma)$ la longueur d'un chemin $\gamma$ et on sait que la distance associée ainsi obtenue est égale à la distance de Kobayashi $k_{D}$. Nous renvoyons le lecteur intéressé par ces questions à [3], [6] et [7].

\section{DÉmonstration DU PREMIER ThÉORÈme}

Nous allons montrer le théorème suivant.

Théorème 3.1. Soit $M_{1}$ une variété complexe connexe taut de dimension n, soit $M_{2}$ une variété complexe connexe de même dimension $n$ dont le revêtement universel est analytiquement isomorphe à un domaine borné strictement convexe de $\mathbb{C}^{n}$. Soit $a \in M_{1}$ et soit $f: M_{1} \longrightarrow M_{2}$ une application holomorphe telle que, pour tout $v \in T_{a}(M)$,

$$
F_{M_{2}}(f(a), T f(a) \cdot v)=F_{M_{1}}(a, v) .
$$

Alors $f$ est un revêtement de $M_{1}$ sur $M_{2}$.

Démonstration. Soit $\pi_{1}: \widetilde{M}_{1} \longrightarrow M_{1}$ le revêtement universel de $M_{1}$. L'application $\pi_{1}$ étant un homéomorphisme local, $\widetilde{M}_{1}$ est naturellement muni d'une structure de variété analytique complexe qui rend $\pi_{1}$ analytique. De même, soit $\pi_{2}: \widetilde{M}_{2} \longrightarrow M_{2}$ le revêtement universel de $M_{2}$. L'application holomorphe $f \circ \pi_{1}: \widetilde{M}_{1} \longrightarrow M_{2}$ d'un espace simplement connexe $\widetilde{M}_{1}$ à valeurs dans $M_{2}$ se relève en une application continue $F: \widetilde{M}_{1} \longrightarrow \widetilde{M}_{2}$. D'autre part, $\pi_{2} \circ F$ est égal à $f \circ \pi_{1}$ qui est une application holomorphe. Par la définition même de la structure de variété analytique complexe sur $\widetilde{M}_{2}$, ceci prouve que $F$ est holomorphe.

D'après S. Kobayashi [7], on sait que $\pi_{1}$ et $\pi_{2}$ sont des isométries pour la métrique infinitésimale de Kobayashi. Soit $b \in \widetilde{M}_{1}$ un point tel que $\pi_{1}(b)=a$. Alors, on a, pour tout $v \in T_{b} \widetilde{M}_{1}$,

$$
F_{\widetilde{M}_{2}}(F(b), T F(b) \cdot v)=F_{\widetilde{M}_{1}}(b, v) .
$$

Comme $\widetilde{M}_{2}$ est analytiquement isomorphe à un domaine borné strictement convexe et que $\widetilde{M}_{1}$, revêtement universel de $M_{1}$, est taut, on déduit du théorème de I. Graham [4] que $F$ est un isomorphisme analytique de $\widetilde{M}_{1}$ sur $\widetilde{M}_{2}$. Si on considère maintenant un ouvert $U$ de $M_{2}$ tel que $\pi_{2}^{-1}(U)$ soit réunion de feuilles $U_{i}$ tel que $\left.\pi_{2}\right|_{U_{i}}: U_{i} \longrightarrow U$ soit un homéomorphisme, il est clair que $f^{-1}(U)$ est réunion d'ouverts $V_{j}$ tels que $\left.f\right|_{V_{j}}: V_{j} \longrightarrow U$ soit un homéomorphisme. Ainsi, $f$ est bien un revêtement.

On peut aussi montrer le théorème suivant. 
Théorème 3.2. Soient $M_{1}$ et $M_{2}$ deux variétés complexes connexes de la même dimension $n$, dont les revêtements universels sont analytiquement isomorphes à des domaines bornés convexes de $\mathbb{C}^{n}$. Soit $a \in M_{1}$ et soit $f: M_{1} \longrightarrow M_{2}$ une application holomorphe telle que, pour tout $v \in T_{a}\left(M_{1}\right)$,

$$
F_{M_{2}}(f(a), T f(a) \cdot v)=F_{M_{1}}(a, v) .
$$

Alors $f$ est un revêtement de $M_{1}$ sur $M_{2}$.

Démonstration. Comme dans la démonstration du théorème précédent, on considère les revêtements universels $\pi_{1}: \widetilde{M}_{1} \longrightarrow M_{1}$ et $\pi_{2}: \widetilde{M}_{2} \longrightarrow M_{2}$ de $M_{1}$ et $M_{2}$. L'application $f$ se relève en une application holomorphe $F: \widetilde{M}_{1} \longrightarrow \widetilde{M}_{2}$. Il nous faut alors montrer que $F$ est un isomorphisme analytique de $\widetilde{M}_{1}$ sur $\widetilde{M}_{2}$. Si $b \in \widetilde{M}_{1}$ est un point tel que $\pi_{1}(b)=a$, alors on a, pour tout $v \in T_{b} \widetilde{M}_{1}$,

$$
F_{\widetilde{M}_{2}}(F(b), T F(b) \cdot v)=F_{\widetilde{M}_{1}}(b, v) .
$$

Comme $\widetilde{M}_{1}$ et $\widetilde{M}_{2}$ sont analytiquement isomorphes à des domaines bornés convexes, on sait d'après L. Lempert [8] et H. Royden et P. Wong [9] (voir aussi M. Jarnicki et P. Pflug [6]) que les métriques infintésimales de Carathéodory et de Kobayashi coïncident sur $\widetilde{M}_{1}$ et $\widetilde{M}_{2}$. On a donc, pour tout $v \in T_{b} \widetilde{M}_{1}$,

$$
E_{\widetilde{M}_{2}}(F(b), T F(b) \cdot v)=E_{\widetilde{M}_{1}}(b, v) .
$$

On peut donc appliquer le théorème 1.1 et $F$ est bien un isomorphisme analytique de $\widetilde{M}_{1}$ sur $\widetilde{M}_{2}$. Le reste de la démonstration est inchangé.

On peut alors se demander si on peut obtenir des généralisations du lemme de Schwarz-Pick; plus précisement, on considère une application $f$ d'une variété complexe $M$ dans elle-même et on suppose qu'il existe un point $a \in M$ tel que, pour tout $v \in T_{a}(M)$, on ait

$$
F_{M}(f(a), T f(a) \cdot v)=F_{M}(a, v) .
$$

Si on suppose vérifiées les hypothèses du théorème 3.1 ou $3.2, f$ est une application de revêtement de $M$ sur $M$ et on peut se demander pour quelles variétés cette hypothèse entraîne que $f$ est un automorphisme analytique de $M$. C'est ce que nous allons faire dans les deux paragraphes suivants, d'abord en dimension 1, ensuite en dimension supérieure.

\section{LE CAS DE LA DIMENSION 1}

Nous allons considérer le cas où $D$ est un domaine borné de $\mathbb{C}$. On sait alors que son revêtement universel est isomorphe au disque-unité $\Delta$ qui est strictement convexe. On peut donc appliquer les résultats précédents et nous allons montrer le théorème suivant.

Théorème 4.1. Soit $D$ un domaine borné de $\mathbb{C}$. Soit $\delta=\inf L(\gamma)$, où $\gamma$ est un lacet de $D$ non homotope comme lacet à un lacet constant. Supposons que $\delta$ soit strictement positif. Soit $a \in D$, et soit $f: D \longrightarrow D$ une application holomorphe telle que, pour tout $v \in T_{a}(D)$, on ait:

$$
F_{D}\left(f(a), f^{\prime}(a) \cdot v\right)=F_{D}(a, v) .
$$

Alors $f$ est un automorphisme analytique de D. 
Démonstration. D'après le théorème $3.1, f$ est un revêtement de $D$ sur $D$. On en déduit que, pour tout $n>0$, l'itérée $f^{n}(=f \circ \ldots \circ f n$ fois) est aussi un revêtement de $D$ sur $D$. L'application $f^{n}$ induit une application $\pi_{1}\left(f^{n}\right)$ entre les groupes d'homotopie $\pi_{1}(D, b)$ et $\pi_{1}(D, f(b))$, et d'après les théorèmes classiques sur les revêtements, $\pi_{1}\left(f^{n}\right)$ est injective (voir par exemple M. Greenberg [5] ou E. Spanier [10]).

Ainsi, si $\gamma:[u, v] \longrightarrow D$ est un lacet de classe $\mathcal{C}^{1}$ par morceaux non homotope à un lacet constant, il en est de même de $f^{n} \circ \gamma$. Par suite, la longueur $L\left(f^{n} \circ \gamma\right)$ est toujours supérieure ou égale à $\delta>0$. Comme $f^{n}$ est une suite bornée, on peut d'après le théorème de Montel en extraire une suite $f^{n_{k}}$ convergeant uniformément sur tout compact de $D$ vers $g$. On a alors

$$
\int_{u}^{v}\left|\left(f^{n} \circ \gamma\right)^{\prime}(t)\right| d t=\int_{u}^{v}\left|\left(f^{n}\right)^{\prime}(\gamma(t))\right|\left|\gamma^{\prime}(t)\right| d t \geq \delta>0 .
$$

Il est clair que $\left(f^{n_{k}}\right)^{\prime}(z) \longrightarrow g^{\prime}(z)$ uniformément sur l'image de $\gamma$ qui est compacte. On en déduit que

$$
\lim \int_{u}^{v}\left|\left(f^{n_{k}}\right)^{\prime}(\gamma(t))\right|\left|\gamma^{\prime}(t)\right| d t=\int_{u}^{v}\left|g^{\prime}(\gamma(t))\right|\left|\gamma^{\prime}(t)\right| d t \geq \delta>0 .
$$

Ceci entraîne que $g^{\prime}(z)$ n'est pas identiquement nul. Ainsi $g$ n'est pas dégénérée au sens de $\mathrm{H}$ Cartan [2], et le théorème 4 de [2] montre que $f$ (et $g$ ) sont des automorphismes analytiques de $D$. Le théorème est démontré.

Remarquons que, si $D$ est égal à $\Delta^{*}=\Delta \backslash\{0\}$, pour tout $n>1$, l'application $f: z \mapsto z^{n}$ est un revêtement de $\Delta^{*}$ sur $\Delta^{*}$ et n'est pas un automorphisme de $\Delta^{*}$. Ainsi, le théorème 4.1 n'est pas vrai pour tout domaine borné de $\mathbb{C}$.

\section{LE CAS DE LA DIMENSION SUPÉRIEURE}

Nous allons nous limiter à traiter le cas d'un domaine borné de $\mathbb{C}^{n}$ dont le premier groupe d'homotopie $\pi_{1}(D, a)$ est isomorphe à $\mathbb{Z}$. Nous avons alors le théorème suivant.

Théorème 5.1. Soit $D$ un domaine borné de $\mathbb{C}^{n}$ tel que son premier groupe d'homotopie soit isomorphe à $\mathbb{Z}$. Soit $f: D \longrightarrow D$ une application holomorphe qui est un revêtement. Pour tout $n \in \mathbb{N}$, soit

$$
\delta(n)=\inf L_{k}(\gamma),
$$

où $\gamma$ parcourt l'ensemble des lacets dont la classe d'homotopie dans $\pi_{1}(D)$ est, en module, égale à $n$. Supposons que $\delta(n)$ tende vers $+\infty$ quand $n$ tend vers $+\infty$. Alors $f$ est un automorphisme analytique de $D$.

Démonstration. L'application $f$ induit un homomorphisme de groupe $\pi_{1}(f): \mathbb{Z} \longrightarrow$ $\mathbb{Z}$. Comme $f$ est un revêtement, $\pi_{1}(f)$ est injectif. C'est donc la multiplication par un nombre entier $a$. Il suffit donc de montrer que $a$ est de module 1 .

Faisons la démonstration par l'absurde. Supposons que $|a|>1$. Soit $\gamma$ est un lacet dont la classe $\alpha$ dans $\pi_{1}(D)$ est non nulle. Comme $f$ est une isométrie pour la métrique infinitésimale de Kobayashi, pour tout $n \in \mathbb{N}$, la longueur $L_{k}\left(f^{n} \circ \gamma\right)$ est égale à la longueur $L_{k}(\gamma)$. D'autre part, la classe d'homotopie de $f^{n} \circ \gamma$ est égale à $a^{n} \alpha$ et, comme $a^{n} \alpha$ tend vers l'infini, la longueur $L_{k}\left(f^{n} \circ \gamma\right)$ qui est supérieure ou égale à $\delta\left(a^{n} \alpha\right)$ tend vers l'infini avec $n$. Contradiction. Le théorème est démontré. 


\section{Remarques et Questions}

On peut faire au sujet du théorème 5.1 la remarque suivante: comme $D$ est un domaine borné de $\mathbb{C}^{n}$, il existe une constante $c>0$ telle que, pour tout $x \in D$, pour tout $v \in \mathbb{C}^{n}, F_{D}(x, v) \geq c\|v\|$. Par suite, pour tout lacet $\gamma, L_{k}(\gamma) \geq c L(\gamma)$, et l'hypothèse que $\delta(n)=\inf L_{k}(\gamma)$ tende vers $+\infty$ est a priori plus faible que la même hypothèse faite en utilisant la longueur de $\gamma$ pour la norme hermitienne.

Il faut aussi remarquer que, comme en dimension 1, le théorème 5.1 n'est pas vrai sans une hypothèse sur $\delta(n)$. En effet, l'application holomorphe

$$
f: \Delta^{*} \times \Delta \longrightarrow \Delta^{*} \times \Delta
$$

définie par $f:\left(z_{1}, z_{2}\right) \mapsto\left(z_{1}^{2}, z_{2}\right)$ est un exemple de revêtement qui n'est pas un automorphisme analytique. On peut même construire des exemples $D$ qui sont égaux à l'intérieur de leur fermeture.

On peut aussi poser un problème semblable en utilisant la métrique infitésimale de Carathéodory. On a alors le problème suivant: soit $D$ un domaine borné de $\mathbb{C}^{n}$. Soit $f: D \longrightarrow D$ une application qui est une isométrie pour la métrique infinitésimale de Carathéodory en un point $a$ de $D$. A quelle condition sur $D, f$ est-elle un automorphisme analytique de $D$ ? Par exemple, M. Jarnicki et P. Pflug [ [6] ont donné une réponse positive lorsque $D$ est une couronne $\{z \in \mathbb{C}|1<| z \mid<r\}$. Il serait intéressant de connaître d'autres cas où on peut démontrer le même résultat.

\section{REFERENCES}

[1] L. Belkhchicha. Caractérisation des isomorphismes analytiques sur la boule-unité de $\mathbb{C}^{n}$ pour une norme. Math. Z. 215 (1994), p. 129-141. MR 94m:32037

[2] H. Cartan. Sur les fonctions de plusieurs variables complexes. L'itération des transformations intérieures d'un domaine borné. Math. Z. 35 (1932), p. 760-773.

[3] T. Franzoni and E. Vesentini. Holomorphic maps and invariant distances. Math. Studies 40, North-Holland, Amsterdam, 1980. MR 82a:32032

[4] I. Graham. Holomorphic mappings into strictly convex domains which are Kobayashi isometries at a point. Proc. Amer. Math. Soc. 105 (1989), p. 917- 921. MR 89k:32048

[5] M. Greenberg. Lectures on algebraic topology. W. A. Benjamin, New-York, (1967). MR 35:6137

[6] M. Jarnicki and P. Pflug. Invariant distances and metrics in complex analysis. De Gruyter Expositions in Mathematics 9, De Gruyter, Berlin, 1993. MR 94k:32039

[7] S. Kobayashi. Intrinsic distances, measures and geometric function theory. Bull. Amer. Math. Soc. 82 (1976), p. 357-416. MR 54:3032

[8] L. Lempert. Holomorphic retracts and intrinsic metrics in convex domains. Anal. Math., 8 (1982), p. 257-261. MR 84f:32026

[9] H. Royden and P. Wong. Carathéodory and Kobayashi metrics on convex domains. Preprint (1983).

[10] E. Spanier. Algebraic topology. McGraw-Hill, New York 1966. MR 35:1007

[11] J.-P. Vigué. Caractérisation des automorphismes analytiques d'un domaine convexe borné. C. R. Acad. Sc. Paris Série I Math., 299 (1984), p. 101-104. MR 85h:32042

[12] J.-P. Vigué. Sur la caractérisation des automorphismes analytiques d'un domaine borné. Portugaliae Math. 43 (1986), p. 439-453. MR 89a:32029

[13] H. Wu. Normal families of holomorphic mappings. Acta Math. 119 (1967), p. 194-233. MR 37:468

Upres A 6086 Groupes de Lie et Géométrie, SP2Mi, Mathématiques, Université de Poitiers, BP 30179, 86962 Futuroscope Cedex, France

E-mail address: vigue@mathlabo.univ-poitiers.fr 